\title{
VLC Modelization for VLC-PLC System: Evaluation of Optical, Electrical, and Frequency Behavior
}

\author{
Yara Yaacoub*, F. Nouvel* $^{*}$ S. Haese*, J.-Y. Baudais ${ }^{\dagger}$ \\ *INSA-Rennes, IETR \\ †Institut d'électronique et des technologies du numérique, IETR, CNRS, Rennes, France
}

\begin{abstract}
The integration of powerline communication (PLC) with visible light communication (VLC) has attracted the attention of many researchers. Cascading these two techniques offers the possibility of performing a wired transmission over existing powerline infrastructure and a last-mile energy-efficient wireless transmission through pre-installed LEDs for indoor illumination. Therefore, VLC should be deeply analyzed to optimize its integration with other systems. So far, most research has only modeled the channel and modulation bandwidth of the VLC system. However, the electrical and optical aspects are rarely taken into account and there is no explicit expression of the received signal as a function of that emitted. So in this paper, An explicit expression is proposed allowing a direct calculation of the photo-current as a function of the transmitted current taking into account the optical, electrical, and frequency behavior of the VLC system. This expression is validated using measurement results.

Index Terms-VLC channel, LED frequency response, optical power, photo-current.
\end{abstract}

\section{INTRODUCTION}

VLC is a relatively new technology based on LEDs. It can transmit on a very large regulation-free bandwidth using intensity modulation and direct detection. Indeed, it is an energy efficient communication system because of the LED capability to support simultaneously communication and lighting [1]. Moreover, VLC is preferred to be used in communicationsensitive indoors like hospitals, as it satisfies electromagnetic compatibility requirements [2]. However, combining VLC with another technology is necessary to increase the network coverage. One of the most suitable technologies is the powerline communication (PLC) because it serves as a natural backbone for the high power LEDs used for illumination [3].

The first hybrid PLC and VLC system prototype was proposed in [4]. It was based on using a single carrier binary phase-shift keying modulation to provide a low rate transmission. Further works studied OFDM to compensate for the effect of multipath fading [5]. Later, many other scenarios were built to optimize the hybrid system in terms of complexity, cost, rate, etc. [6]-[8].

In hybrid communication, there are two types of relaying systems: $i$ ) the decode and forward (DF), ii) the amplify and forward (AF). The DF technique consists on decoding the PLC signal and re-encoding it before being transmitted by the VLC channel. Whereas, in the AF the PLC signal is amplified and re-transmitted as it is by the optical system.
This technique is less complicated but, at the same time, it can create a big challenge [7]. More precisely, when VLC is cascaded to a highly attenuated system like PLC, the available modulation bandwidth of the whole system is reduced. This is owing to the limited frequency response of the LED (few $\mathrm{MHz}$ ) which can lead to a serious communication failure [2]. Therefore when adopting AF technique, the parameters of the VLC system should be well adjusted and its components should be carefully chosen to ensure successful PLC-VLC communication. However, the LED modulation bandwidth varies according to the technology adopted for generating a white light. Actually, in lighting industry, there are two commonly used techniques: the first one uses a blue LED with a yellow phosphor coat. This coat has a very slow response that reduces the modulation bandwidth up to $1 \mathrm{MHz}$. The second technique is based on combining three colors: red, green, and blue (RGB LEDs). The RGB LEDs provide a larger bandwidth than that of the phosphor LED. However, balancing their intensities to get the desired white color can be complicated in manufacturing [2].

Unlike VLC, PLC technology has already been deployed and many standards have been elaborated like HomePlug 1.0.1 (2005), HomePlugAV (2005-2007), HD-PLC (2007), IEEE 1901 (2010), G3-PLC (2010) or HomePlugAV2 (2010) [9].

Our objective is to be able to transmit HomePlugAV-like signals through a trichromatic lighting system (commercial RGB LEDs) using the AF method. But as explained before, when adopting AF, the already attenuated PLC signal is remarkably affected by the VLC system. So, a preliminary study of the VLC system should be made. Unfortunately, there is no explicit expression making it possible to evaluate the received VLC signal as a function of the transmitted signal taking into account all of the optical, electrical and frequency behavior of the system. In fact, most research has only modeled the VLC channel [2], [10]. In [11] the optical and electrical nonlinearity of LEDs is investigated. However, the spectral nonlinearity of the photo-current generated at the receiver is not taken into account. Further, in [12] the optical side of the VLC system is covered without considering the effect of the LED optical nonlinearity. In [13], a theoretical analysis of the relationship between the current energy of the hardware circuit and the optical power is performed taking into account all nonlinearties (optical, electrical and frequency) of the VLC 
system. Due to the complexity of this model, an explicit expression to simplify the calculation of the received signal is not obtained. However, This expression is very important for a quick evaluation of the hardware system. Thus, in our paper, an attempt is made to compensate for these shortcomings. Consequently, an explicit expression permitting the calculation of the photo-current as a function of the transmitted signal is proposed. This expression takes into account the electrical, optical and frequency behavior of the VLC system in order to built and optimize PLC-VLC system.

The remainder of this paper is organized as follow: In Section II, VLC channel is discussed and the LED frequency response expression is also figured out based on experimental results. In Section III-A, an analytical expression is provided to describe the relationship between the maximum transmitted and generated photo-current. Measurements are carried out in Section III-B to validate the accuracy of this expression. In Section III-C, a comparison was made between the measurement results and the values obtained using our model with and without taking into account the effect of the optical nonlinearity and the LED spectral width. Finally, conclusions are given in Section IV.

\section{VLC CHANNEL MODEL}

The VLC channel is actually composed from a direct line of sight (LOS) and a non direct line of sight (NLOS). A NLOS has a very low power compared to the LOS. According to [14], the NLOS can be neglect and the channel model considers only the LOS. Hence, the channel is represented by the following expression:

$$
H_{C}= \begin{cases}\frac{(m+1) A \cos ^{m}(\Phi) \cos (\psi)}{2 \pi D^{2}} G, & \psi<\psi_{\mathrm{FOV}} \\ 0 & \psi>\psi_{\mathrm{FOV}}\end{cases}
$$

where $m$ is the Lambertian index, $A$ is the active area of the photodiode, $\Phi$ and $\psi$ are the angles of irradiance and incidence respectively, $D$ is the distance between the LED and the photodiode, $\psi_{\mathrm{FOV}}$ is the irradiance angle of the field of vision of the receiver and $G$ is the gain of the lens that concentrates the light to the photodiode. Moreover, the LED limited bandwidth is an important factor that can affect the VLC and the hybrid channel as well. Hence, the overall system attenuation can be expressed as follows:

$$
H_{V}=H_{L} H_{C}
$$

where $H_{L}$ is the LED frequency response (LFR).

In order to model the attenuation of the VLC system, the LFR should be measured and then modeled. Hence, a wideband VLC transmitter was manufactured. The latter consists of monochromatic LED (red, green or blue) from the RGB module, LUXEON MultiColor LED Module 2.5 W [15], and a wide-band bias tee circuit up to $100 \mathrm{MHz}$ at $-3 \mathrm{~dB}$. Figure 1a illustrates the realized bias tee which is a three-port network used to set the DC bias point of certain electronic components without disturbing other components. The equivalent circuit model is represented in Figure $1 \mathrm{~b}$ where the low-frequency

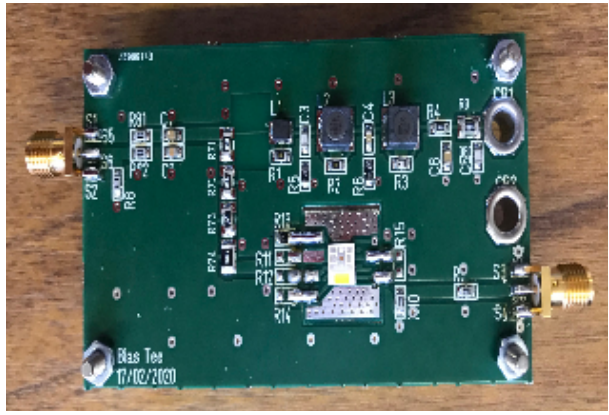

(a)

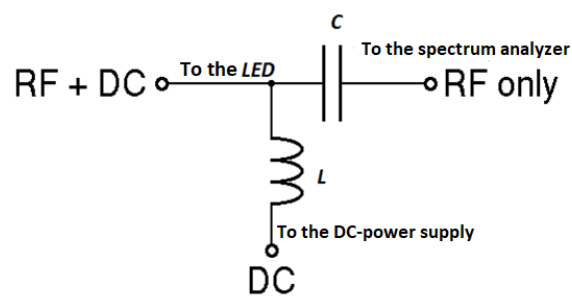

(b)

Figure 1: Bias Tee. (a) Our manufactured circuit, (b) explanatory scheme.

port is used to define the polarization; the high-frequency port passes the data signals but blocks bias levels; the combo port is connected to the device, which can provide both polarization and data signals [16] (see Figure 1b). Back to LFR measurements, in our study, each LED are biased at $100 \mathrm{~mA}$. As shown in Figure 2, the red LED has the widest modulation band of $20 \mathrm{MHz}$ at $-3 \mathrm{~dB}$. However, the green and blue LEDs have approximately the same response with a $-3 \mathrm{~dB}$ cutoff frequency of $5 \mathrm{MHz}$. Hence, the LFR can be modeled by a first order low-pass filter:

$$
H_{L}(f)=\frac{1}{1+j \frac{f}{f_{c}}}
$$

where $f_{c}$ is the $-3 \mathrm{~dB}$ cutoff frequency of the LED LFR. Therefore, the red LED is chosen to perform the first PLCVLC transmission tests, and thus it is used to do the expression validation measurement.

\section{ANALYTIC EXPRESSION OF THE TRANSMITTED CURRENT}

\section{A. Proposed model}

When dimensioning and optimizing a PLC-VLC system, it is important to have an estimate of the maximum current amplitude that the photodiode would generate when transmitting signals through the LED, without the need to repeat the measurements each time the amplitude of the transmitted signal changes. Thus, an analytical relationship between the transmitted and the generated currents must be found. In this section, our proposal is described and its accuracy is validated 


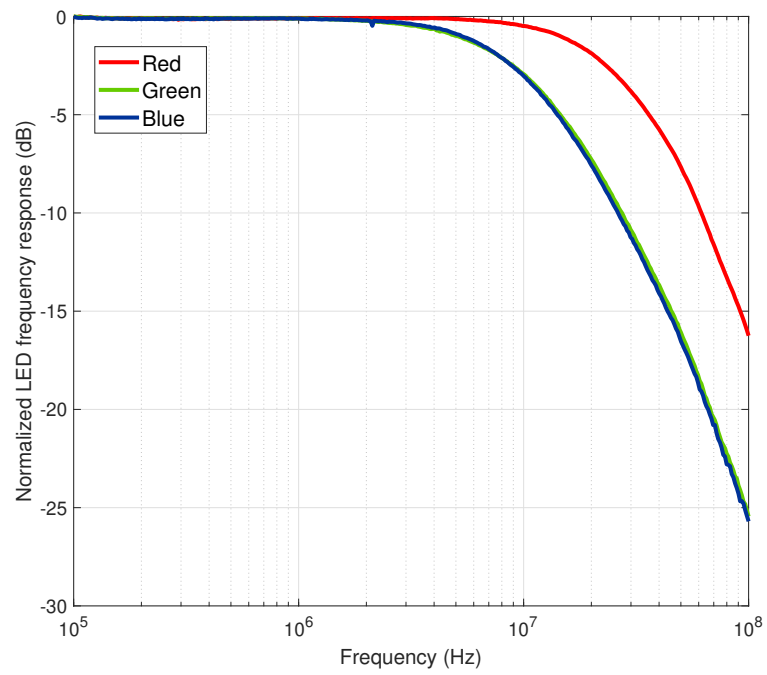

Figure 2: Red, green, and blue LED frequency responses.

by measurement results. In LED datasheet, the typical transmitted power density at the LED's dominant wavelength $P$ is not given explicitly. Although, all the curves presented in the LED data sheet are normalized to this value. However, it can be calculated using the luminous flux expression [17] as following:

$$
P=\frac{\phi}{V_{\max } \int_{380}^{780} v(\lambda) p(\lambda) d \lambda}
$$

where $\phi$ is LED the luminous flux, $v(\lambda)$ is normalized to its maximum eye efficacy $V_{\max }\left(V_{\max }=683 \mathrm{~lm} / \mathrm{W}\right.$ at $\lambda=$ $555 \mathrm{~nm}$ ) and $p(\lambda)$ is the optical power density normalized to $P$. $\phi$ and $p(\lambda)$ are given in the LED datasheet. To be able to calculate the integral, $p(\lambda)$ and $v(\lambda)$ are modeled by the sum of two Gaussian functions [13]:

$$
p(\lambda)=a e^{-\left(\frac{\lambda-b}{c}\right)^{2}}+d e^{-\left(\frac{\lambda-e}{f}\right)^{2}}
$$

and

$$
v(\lambda)=a_{1} e^{-\left(\frac{\lambda-b_{1}}{c_{1}}\right)^{2}}+d_{1} e^{-\left(\frac{\lambda-e_{1}}{f_{1}}\right)^{2}}
$$

Moving to the relationship between the light output and the current crossing the LED $L(i)$, it is given in the datasheet normalized to its typical and it could be expressed by a polynomial function as following:

$$
L(i)=k_{1} i^{3}+k_{2} i^{2}+k_{3} i+k_{4}
$$

So, the LED transmitted power density in function of wavelength at a determined current can be described by:

$$
P_{\mathrm{tr}}(\lambda, i)=\operatorname{Pp}(\lambda) L(i)
$$

However, this transmitted power is affected by the channel attenuation and the LFR. Hence, the received power density is written as follows:

$$
p_{\text {rec }}(\lambda, i)=P_{\text {tr }} H_{V}
$$

The photodiode receives the light and generates the photocurrent depending on its sensitivity. The following equation shows the current spectral density as a function of the received optical power density:

$$
i_{P}(\lambda, i)=S s(\lambda) p_{\mathrm{rec}}(\lambda, i)
$$

where $S$ is the maximum sensitivity of the photodiode, $s(\lambda)$ is given in the photodiode's datasheet. In order to reduce the complexity of calculation in the following steps, we modeled only the part of the spectrum where the RGB LEDs radiate, as follows:

$$
s(\lambda)=s_{1} \lambda^{2}+s_{2} \lambda+s_{3}
$$

Hence, the overall generated current is the integral of the current density over the all spectrum:

$$
I(i)=\int_{380}^{780} i_{P}(\lambda, i) d \lambda
$$

where the integration boundries are the extremums of the visible spectrum. However, since the boundaries of $p(\lambda)$ are too attenuated for wavelengths greater than $780 \mathrm{~nm}$ and less than $380 \mathrm{~nm}$. the integral of $I(i)$ can be considered from 0 to $+\infty$ to simplify the final expression The final expression of the generated current is:

$$
\begin{aligned}
I(i)= & P H_{B} H_{V} S L(i) \int_{0}^{+\infty} s(\lambda) p(\lambda) d \lambda \\
= & P H_{B} H_{V} S L(i) \sqrt{\pi}\left(c a\left(s_{1}\left(\frac{c^{2}}{2}+b^{2}\right)+s_{2} b+s_{3}\right)\right. \\
& \left.\left.+d f\left(s_{1}\left(\frac{f^{2}}{2}+e^{2}\right)+s_{2} e+s_{3}\right)\right)\right)
\end{aligned}
$$

where $H_{B}$ is the bias tee frequency attenuation. $P, H_{V}$ and $L(i)$ are respectively the typical power, the VLC channel, and the light output described in (4), (2), and (7). The expression results has been compared to measurements performed on our testbed described in Section II.

\section{B. Current measurement setup}

In this part we validate (13) using the red LED of the previously described transmitter in Section II. The LED is biased at $110 \mathrm{~mA}$, the receiver used involves the BPW34 photodiode [18] and a trans-impedance amplifier (TIA) of $50 \mathrm{k} \Omega$. The transmitter and the receiver are aligned and separated by a distance of $13 \mathrm{~cm}$. The $50 \mathrm{kHz}$ transmitted current has a maximum amplitude of $10 \mathrm{~mA}$. The bias tee attenuation at this frequency is 0.6 . The measurement result shows that the maximum voltage amplitude at the output of TIA amplifier is $10 \mathrm{mV}$ which means that the maximum amplitude of the generated photo-current is $200 \mathrm{nA}$.

\section{Results analysis}

As Figs. 3a to 3c show, the proposed model of $p(\lambda)(5)$, $L(i)$ (7) and $s(\lambda)$ (11) fits properly the curves of the LED and photodiode datasheets. In order to show the improvement brought by this model when it takes into account the optical nonlinearity $L(i)$ and the LED spectral power width $p(\lambda)$ with 
Table I: comparison between $V_{T I A}$ calculated in 3 different cases: considering all the non-linearities, considering $L(i)$ linear, and considering $s(\lambda)$ and $p(\lambda)$ at the dominant wavelength of the LED .

\begin{tabular}{|c|c|c|c|}
\hline Assumption & $I(n A)$ & $V_{T I A}(m V)$ & $E R R(\%)$ \\
\hline i) proposed model & 244 & 12.2 & 22 \\
\hline ii $L(i)$ linear & 281 & 14.1 & 41 \\
\hline iii) $p(\lambda)$ and $s(\lambda)$ at $\lambda_{\text {dominant }}$ & 254 & 12.7 & 27 \\
\hline
\end{tabular}

the spectral sensitivity of the photodiode $s(\lambda)$. The maximum generated photocurrent $I$ and the TIA's output voltage $V_{T I A}$ were calculated based on the test-bed parameters described in Section III-B at three different cases: $i$ ) when all nonlinearities are considered (our proposed model), $i$ ) when the light output $L(i)$ is considered linear, iii) when the photodiode sensitivity $s(\lambda)$ and $p(\lambda)$ are considered only at the dominant wavelength ( $\lambda_{\text {dominant }}=625 \mathrm{~nm}$ in the case of the LED red). The results in Table I show the precision enhancement in calculation that our model provides in comparison to the results obtained in the last two cases. The calculated $V_{T I A}$ is compared to the measurement $(10 \mathrm{mV})$, resulting in the relative error ERR. It has decreased from $41 \%$ in case $i i$ and $27 \%$ in case iii to $22 \%$ in case $i$. The $22 \%$ error obtained in our model is acceptable regarding the test-bed characteristics. In fact, during measurements, the junction temperature cannot be controlled despite the great influence of the junction temperature on LED characteristics. Especially on the red LED which seems the most affected by the increase in junction temperature [15]. In fact, high power LEDs used for illumination run at high drive current leading to high junction temperature which cause signal power degradation over the time [19]. However, the thermal effect is not treated in this paper. Thus, this simple expression provides a good estimate of the maximum current amplitude that a photodiode could generate, which can help when dimensioning the PLC-VLC system to maintain the linearity of the receiver and not end up being saturated.

\section{CONCLUSION}

In this paper, the optical, electrical, and frequency aspects of the VLC system are studied. The optical channel model is detailed by taking into consideration the LFR. The relationship between the transmitted and the generated current is described by an explicit expression. The efficacy of this expression is tested by a VLC system manufactured in our lab using the red LED of the RGB module. The precision improvement of the model is highlighted by comparing calculation results with and without considering the optical nonlinearity and the LED spectral width. Therefore, a VLC system model is proposed replicating as much as possible its actual optical, electrical, and frequency behavior. A simple expression is established allowing a fast calculation of the photo-current. This expression facilitates the dimensioning and the optimization of the VLC system, especially when it is going to be integrated with other systems like PLC.

\section{ACKNOWLEDGMENT}

The authors would like to thank the Direction générale de l'armement maîtrise de l'information (DGA-MI) for its general supervision and financial support.

\section{REFERENCES}

[1] X. Bao, X. Zhu, T. Song, et al., "Protocol design and capacity analysis in hybrid network of visible light communication and OFDMA systems", IEEE Transactions on Vehicular Technology, vol. 63, no. 4, pp. 1770-1778, 2014.

[2] W. Ding, F. Yang, H. Yang, et al., "A hybrid power line and visible light communication system for indoor hospital applications", Computers in Industry, vol. 68, pp. 170-178, 2015.

[3] S. Nlom, A. Ndjiongue, K. Ouahada, et al., "A simplistic channel model for cascaded PLC-VLC systems", in IEEE International Symposium on Power Line Communications and its Applications, Madrid, Spain, Apr. 2017, pp. 1-6.

[4] T. Komine and M. Nakagawa, "Integrated system of white LED visible-light communication and powerline communication", IEEE Transactions on Consumer Electronics, vol. 49, no. 1, pp. 71-79, 2003.

[5] T. Komine, S. Haruyama, and M. Nakagawa, "Performance evaluation of narrowband OFDM on integrated system of power line communication and visible light wireless communication", in International Symposium on Wireless Pervasive Computing, Phuket, Thailand, 2006, pp. 1-6.

[6] Y. Wang, Y. Wang, N. Chi, et al., "Demonstration of 575-Mb/s downlink and 225-Mb/s uplink bi-directional SCM-WDM visible light communication using RGB LED and phosphor-based LED", Optics express, vol. 21, pp. 1203-1208, Jan. 2013.

[7] M. Mossaad, S. Hranilovic, and L. Lampe, "Amplifyand-forward integration of power line and visible light communications", in IEEE Global Conference on Signal and Information Processing, Orlando, FL, USA, 2015, pp. 1322-1326.

[8] I. Mapfumo and T. Shongwe, "Performance comparison of FFT and DHT hybrid PLC-VLC systems based on DCO-OFDM in impulsive noise", in International Conference Radioelektronika, Bratislava, Slovakia, Slovakia, 2020, pp. 1-6.

[9] A. V. Laere, "Powerline communication transmission performance study in field conditions: Cases of G3PLC for smart metering and Homeplug-AV2 for railway signaling", $\mathrm{PhD}$ thesis, Université de Mons, 2019.

[10] S. Nlom, A. R. Ndjiongue, and K. Ouahada, "Cascaded plc-vlc channel: An indoor measurements campaign", IEEE Access, Apr. 2018.

[11] M. Mossaad, S. Hranilovic, and L. Lampe, "Visible light communications using OFDM and multiple LEDs", IEEE Transactions on Communications, vol. 63, no. 11, pp. 4304-4313, 2015. 


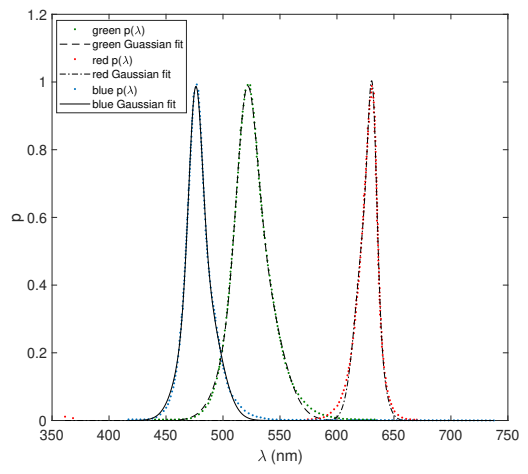

(a)

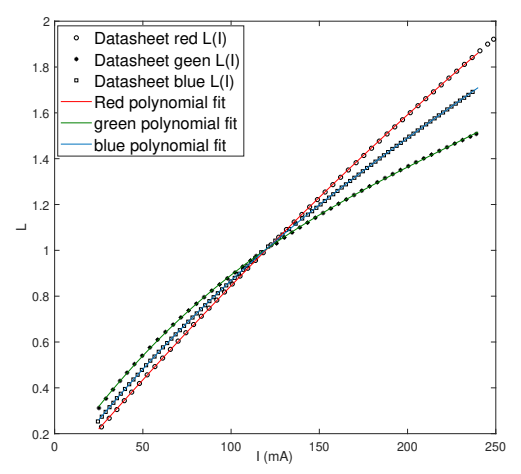

(b)

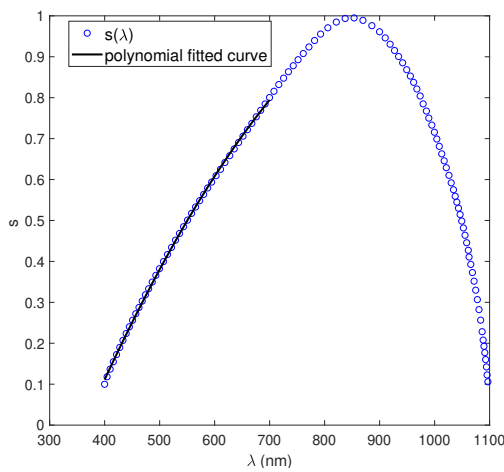

(c)

Figure 3: LED and photodiode datasheet curves models. (a) The spectral power density of red, green, and blue LEDs compared to their modeled curves, (b) the three LEDs' electrical/optical characteristics compared to our polynomial model, (d) spectral sensitivity response of the photodiode compared to our polynomial model.

[12] H. Chun, S. Rajbhandari, G. Faulkner, et al., "Led based wavelength division multiplexed $10 \mathrm{gb} / \mathrm{s}$ visible light communications", Journal of Lightwave Technology, vol. 34, no. 13, pp. 3047-3052, 2016.

[13] L. Zhou, C. Wang, A. Al-Kinani, et al., "Visible light communication system evaluations with integrated hardware and optical parameters", IEEE Transactions on Communications, vol. 66, no. 9, pp. 4059-4073, 2018.

[14] T. Komine and M. Nakagawa, "Fundamental analysis for visible-light communication system using LED lights", IEEE Transactions on Consumer Electronics, vol. 50, no. 1, pp. 100-107, 2004.

[15] Luxeon multicolor module 2.5W, DS244, Lumileds, Apr. 2020.

[16] T. Stratil, P. Koudelka, J. Jankovych, et al., "Broadband over visible light: High power wideband bias-t solution", in International Symposium on Communication Systems, Networks and Digital Signal Processing, Prague, 2016, pp. 1-5.

[17] A. Jabban, S. Haese, and M. Hélard, "Theoretical and experimental optimization of DMT-based visible light communication under lighting constraints", EURASIP Journal on Wireless Communications and Networking, vol. 2020, pp. 1-29, Dec. 2020.

[18] Silicon PIN photodiode, 81521, Vishay, Jan. 2019.

[19] A. Thomas, A. Nair, and P. Student, "Experimental study on the effect of junction temperature on power led's", International Journal of Current Engineering and Scientific Research, vol. 2, no. 9, pp. 14-19, Aug. 2015. 